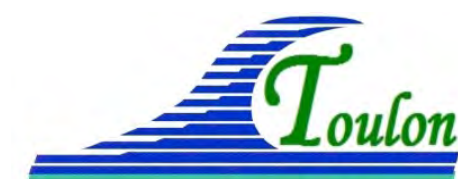
XIV èmes Journées Nationales Génie Côtier - Génie Civil Toulon, 29 juin au $1^{\text {er }}$ juillet 2016

DOI:10.5150/jngcgc.2016.044 (C) Editions Paralia CFL

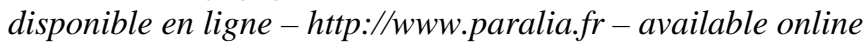

\title{
Outils innovants pour le monitoring des fonds marins : bathymétrie, sédiment, végétations sous-marine et ressources halieutiques par fusion multi-capteurs
}

\author{
Claire NOEL ${ }^{1}$, Eric BAUER ${ }^{1}$, Simon MARCHETTI ${ }^{1}$, \\ Jean-Marc TEMMOS ${ }^{1}$, Michel COQUET ${ }^{1}$
}

\author{
1. SEMANTIC TS. Bureau d'Etudes en Océanographie Acoustique, \\ 1142 Chemin de St Roch, 83110 Sanary s/Mer, France. \\ noel@semantic-ts.fr
}

\section{Résumé :}

Cet article présente le résultat de travaux de recherche menés depuis 2004, dans le domaine de la cartographie 3D et du monitoring de la colonne d'eau, des végétations, des fonds marins (topographie et nature) et sous-sols sédimentaires. Dans ce cadre, nous avons développé des méthodes innovantes de monitoring des petits fonds marins ou plus généralement aquatiques, par fusion des informations des données acoustiques provenant de différents appareillages sonars, opérés simultanément ou non. Ces systèmes fonctionnent à différentes fréquences et apportent donc des informations complémentaires sur le milieu marin.

Les techniques de monitoring, mises au point par SEMANTIC TS reposent sur trois axes de développement présentés dans cet article :

a) Design et conception de plates-formes de sondage légères

b)Fusion de données à partir de différents capteurs et à différentes fréquences

c) Mise au point d'une méthode acoustique de classification pour la nature des fonds \& suite logicielle dédiée (SACLAF SIVA) ainsi que les résultats de leur application aux petits fonds côtiers de Méditerranée.

Mots-clés : Génie côtier, GIZC, Hydraulique maritime, Sédiments, Travaux maritimes, Environnement littoral, Ecosystèmes côtiers, Classification acoustique, SACLAF.

\section{Introduction}

SEMANTIC TS, bureau d'études en océanographie acoustique, utilise le son pour inférer et monitorer l'environnement aquatique : colonne d'eau (ressources halieutiques), végétations (Posidonies, zostères, cymodocées, laminaires, ...), fond (topographie, nature, espèces : moules, crépidules, ...), sous-sol. Si produire des cartes précises des fonds marins est un challenge, assurer leur reproductibilité est encore plus complexe. Or c'est la condition requise, puisque c'est l'évolution entre deux cartes qui fournit des informations pertinentes pour qualifier les changements. Dans ce but, nous développons depuis 2004, au travers de contrats de R\&D pour le compte de la 
DGA/MRIS et de l'Agence de l'eau RMC, une méthode innovante de cartographie et de monitoring par fusion multi-capteurs qui repose sur trois axes de développement :

a) Design de plate-formes de sondage légères

b) Fusion de données à partir de différents capteurs et à différentes fréquences

c) Mise au point d'une méthode acoustique de classification pour la nature des fonds \& suite logicielle dédiée (SACLAF SIVA)

La méthode mise au point exploite les données acquises par plusieurs systèmes sonars fonctionnant à différentes fréquences et apportant donc des informations complémentaires sur le milieu marin. En augmentant le nombre (et la nature) des capteurs, l'ensemble des informations collectées permet une meilleure compréhension du milieu exploré. De la même façon, le médecin utilise des systèmes complémentaires d’imagerie médicale pour surveiller le corps humain : scanner, IRM, radiographie...

\section{Matériel et méthodes}

\subsection{Design de plates-formes légères de sondage multi-capteurs}

Afin de pouvoir opérer ces systèmes de sondage, simultanément, et disposer de données de qualité pour des coûts optimisés, SEMANTIC TS a tout d'abord travaillé sur le design et la conception de plates-formes légères d'acquisition des données en provenance de différents systèmes sonars, d'un GPS et d'une centrale d'attitude. Deux mini-navires océanographiques (de longueur $6.5 \mathrm{~m}$ et $4 \mathrm{~m}$, voir figure 1) ont été conçus, permettant d'opérer 24h/24 un sondeur multifaisceaux, un sonar latéral de coque et un sondeur mono-faisceau de classification. Ces mini-laboratoires naviguant permettent la collecte rapide de grandes quantités de données extrêmement précises et résolvantes (i.e. fournissant des images géo-référencées admettant des pixels de petite taille).

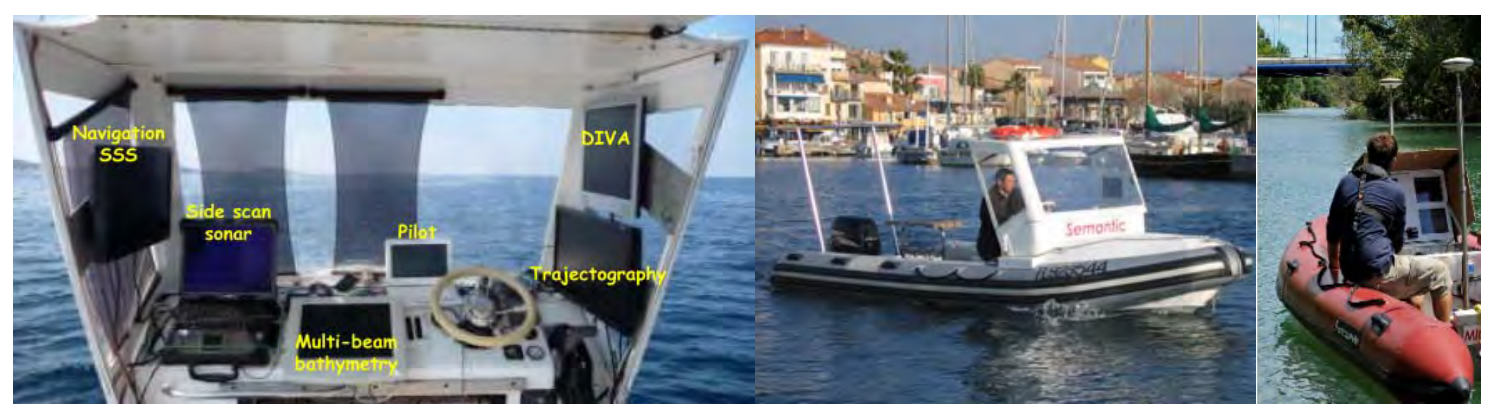

Figure 1. Plates-formes légères de sondage multi-capteurs.

Les coûts de mise en œuvre de ces appareillages, ont été drastiquement réduits par l'usage de ces mini-plates-formes et de ce fait, les méthodes de collecte simultanée de données en provenance de plusieurs capteurs complémentaires, mises au point par SEMANTIC TS et autrefois uniquement réservées au monde militaire ou de la recherche, et à partir de gros navires, sont à présent accessibles au monde civil. Notons 


\section{XIV ${ }^{\text {èmes }}$ Journées Nationales Génie Côtier - Génie Civil \\ Toulon, 29 juin au $1^{\text {er }}$ juillet 2016}

que le navire SEMANTIC et ses équipements (sondeur interférométrique) ont été utilisés en 2007 par l'IFREMER, lors de la phase de validation des performances des capteurs qui ont ensuite été intégrés sur la vedette HALIOTIS en 2008.

\subsection{Acquisition des données multi-capteurs}

Pour cartographier et monitorer les fonds marins (voir le principe schématisé sur la figure 2), on utilise tout d'abord une méthode de type surfacique qui permet d'obtenir une image multi-faisceaux (imagerie sonar latéral) et de contourer sur cette image des zones de réponses acoustiques homogènes. Ces zones sont ensuite "classifiées" à l'aide d'un écho-sondeur. Celui-ci émet du son à la verticale du navire, puis le signal sonore réfléchi par le fond est analysé. Ceci permet d'extraire des caractéristiques du fond qui aident à la classification. Pour cela il est nécessaire de disposer d'un écho-sondeur qui enregistre et restitue le signal réfléchi.

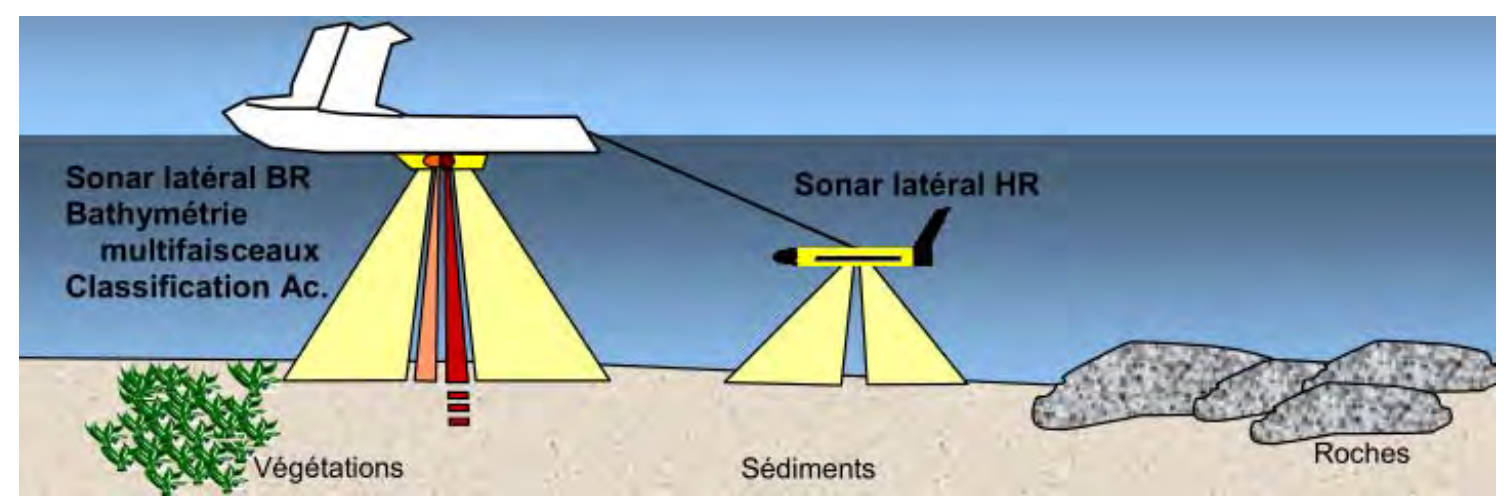

Figure 2. Principe de la méthode d'acquisition multi-capteurs de SEMANTIC TS.

Les données du sonar tracté peuvent être récalées à l'aide de celles du sonar de coque Les données en provenance de différents instruments sont très précisément géoréférencées par le même système de positionnement DGPS RTK/Centrale d'inertie (précision centimétrique) et sont synchronisées sur la même base de temps. Nous avons pour cela, mis au point, un logiciel superviseur innovant, dont le rôle est de cadencer les mesures : acquisitions en provenance des différents appareillages, communications avec la station d'acquisition et gestion des synchronisations, enregistrement des données dans la même base de temps et le même référentiel géodésique. Un SIG (Système d'Information Géographique) scientifique innovant a été développé intégrant le traitement des différentes données acoustiques et la fusion de leurs informations. Celleci est possible et performante car les données, bien qu'en provenance de divers systèmes, sont traitées afin d'être enregistrées dans le même référentiel spatial et temporel. 


\subsection{Méthode acoustique innovante de classification des fonds marins}

Des traitements spécifiques du signal sont ensuite effectués sur la réponse impulsionnelle afin d'en déduire de façon automatisée les caractéristiques géoréférencées de la végétation fixée (présence, absence, hauteur de la canopée) ou du sédiment superficiel (sable fin ou grossier, vase, roche...). Ces traitements ont été mis au point et implémentés dans notre Système Acoustique de CLassification Automatique des natures de Fonds (SACLAF) innovant appelé SIVA (Système d'Inspection Verticale Acoustique) permettant le monitoring simultané des échos dans la colonne d'eau et fournissant une estimation de la biomasse présente dans la colonne d'eau, de la nature, de la profondeur et de la couverture végétale du fond.

Les informations de sorties du système SACLAF sont les suivantes :

a) Type de substrat : Sable fin, sable grossier, vase, roche. Si substrat est intermédiaire le système fournit une classification acoustique correspondant à un milieu équivalent.

b) Présence ou absence de végétation. Hauteur de la canopée (travaux en cours).

c) Nombre de détections halieutiques dans la colonne d'eau

d) Bathymétrie mono-faisceau

La suite logicielle permet d'incorporer des vérités terrain afin de prendre en compte la calibration. La suite logicielle permettant le traitement et la classification des signaux est opérationnelle ; elle est utilisée par les 13 navires du SHOM depuis 2 ans. Le SACLAF peut être utilisé seul ou en fusion avec des données surfaciques.

Les figures qui suivent (voir figure 3) illustrent les résultats des acquisitions et traitements des données de chaque appareillage pris séparément, sur une zone côtière de 500 par 300 m environ.

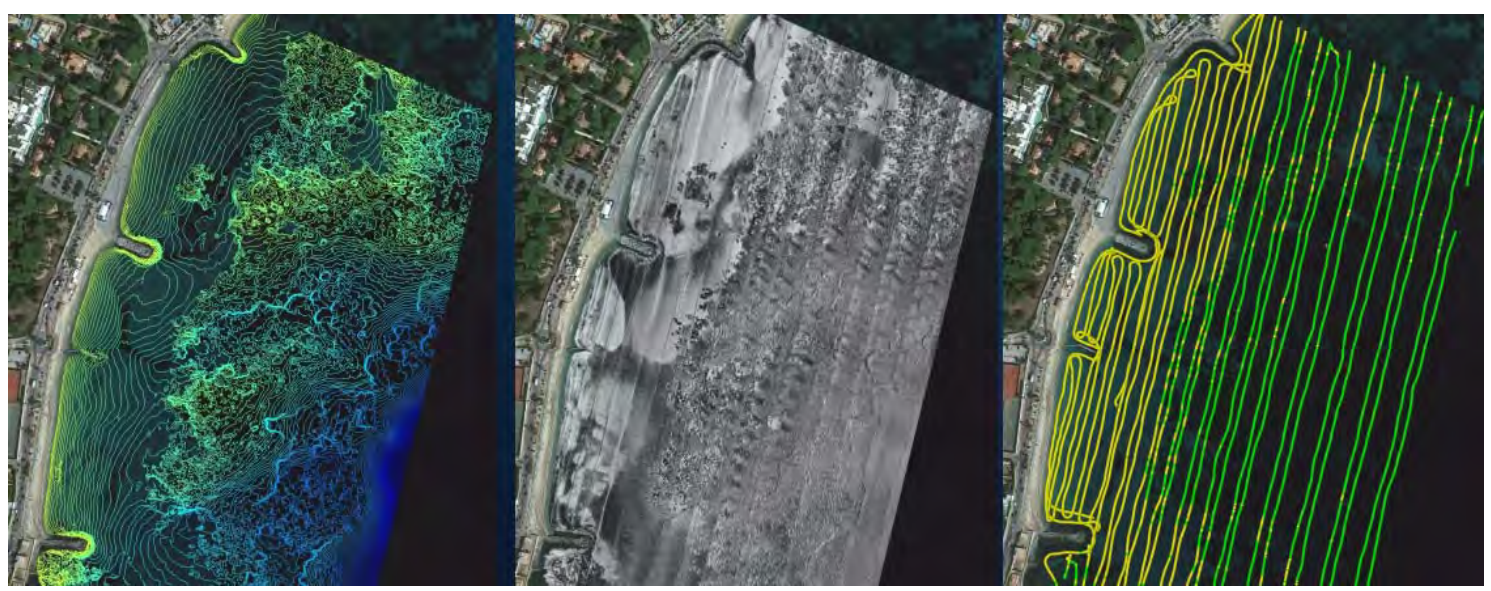

Figure 3. De gauche à droite: Isobathes (micro-rugosité bathymétrique) - Imagerie sonar latéral - SACLAF (Détection herbier en vert - Sédiment en jaune). Taille des zones : $500 \mathrm{~m}$ par $300 \mathrm{~m}$. 


\section{XIV èmes Journées Nationales Génie Côtier - Génie Civil \\ Toulon, 29 juin au $1^{\text {er }}$ juillet 2016}

\subsection{Méthode de monitoring par fusion multi-capteurs}

SEMANTIC TS réalise ensuite la fusion des informations provenant de différents capteurs, à savoir :

a) La bathymétrie $3 D$ renseignant sur la topographie du fond obtenue en même temps que l'imagerie sonar latéral de coque.

b)La micro-rugosité bathymétrique (extraite de la bathymétrie fine, c'est-à-dire haute résolution) fournissant des informations sur la couverture végétale du fond. En effet, en dehors de la précision fournie qui permet d'obtenir la bathymétrie fine, le respect des méthodes d'acquisition (précision de la centrale-GPS et correction des profils de célérité) permet d'obtenir des cartographies de la micro-rugosité du fond. Celle-ci constitue un élément essentiel, car très informatif, de la méthode de fusion multicapteurs. Elle n'est accessible que si la chaine des précisions d'acquisition est respectée à l'échelle centimétrique. Elle découle d'un travail de mesure "propre", ne nécessite pas de contrainte supplémentaire de celles du sonar latéral (qui fournit déjà la bathymétrie multi-faisceaux en sus) et n’a pas de coût supplémentaire.

c) L'imagerie sonar latéral, le niveau de gris renseignant sur la réflectivité et donc sur la nature de la couverture (végétale ou non) du fond.

d) Les informations issues du SACLAF SIVA : présence ou d'absence de végétation (méthode DIVA), nature des fonds sédimentaires (CHIVERS et al., 1990 ; NOEL et al., 2005), estimation de biomasse dans la colonne d'eau.

\section{Résultats}

Nous présentons ici quelques résultats typiques qui permettent d'évaluer la qualité des données obtenues et la performance de la méthode développée.

\subsection{Calibration de nos méthodes}

Nous avons procédé sur plusieurs zones de test à des mesures de calibration des surfaces mesurées au sonar latéral par plongée sous-marine avec un mètre linéaire plombé. Les diamètres des tâches de posidonies ont été mesurés sous l'eau et comparés aux mesures issues de l'imagerie sonar latéral et de la micro-rugosité bathymétrique (voir figure 4). Cette calibration a permis de valider l'excellente adéquation entre les données acoustiques et les données de vérité terrain, et de mettre en évidence l'intérêt de la méthode acoustique, du fait de son fort pouvoir couvrant. 

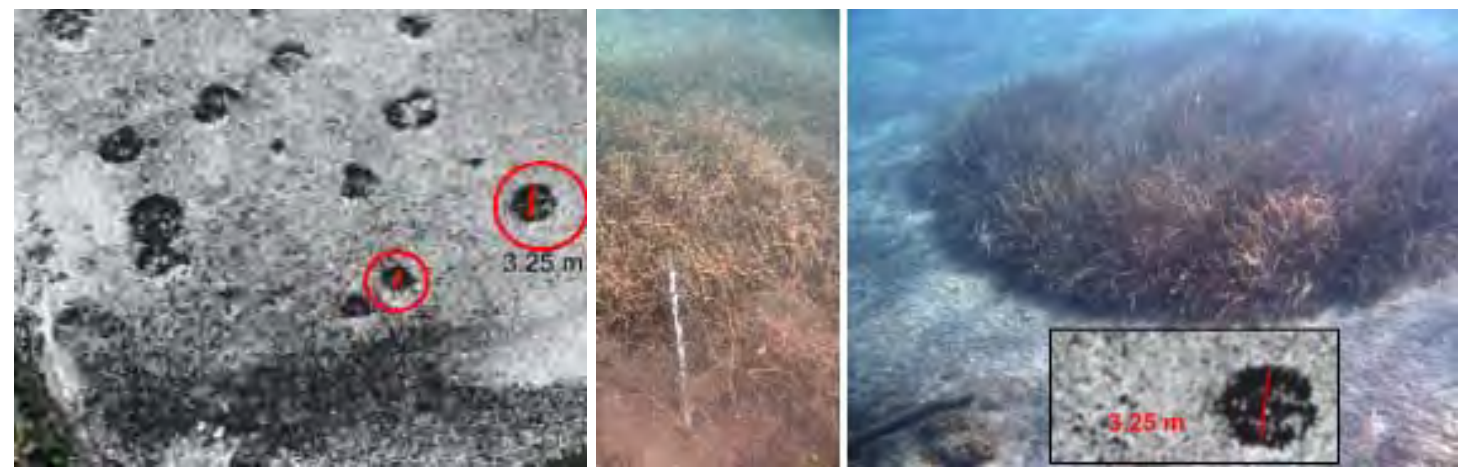

Figure 4. Calibration sonar \& Vérité terrain (Utilisation d'un mètre linéaire métallique). Diamètre mesuré en plongée : $3.25 \mathrm{~m}$

\subsection{Méthode de traitement des données}

Les zones levées étant vastes, nous avons ensuite mis au point une méthode de traitement automatique des images de la micro-rugosité bathymétrique et de l'imagerie sonar latéral. Le traitement des images permet de "géo-contourer" les variations et d'exporter les contours géo-référencés. Cela permet de dresser rapidement et précisément des cartes de biocénoses présentes sur le fond.

\subsection{Méthode de fusion multi-capteurs}

La méthode de fusion multi-capteurs permet de fusionner l'ensemble des informations, surfaciques pour le contourage des zones et linéaires pour la classification, comme l'illustre la figure 5, ainsi que l'extrait représenté en 3D sur la figure 6.

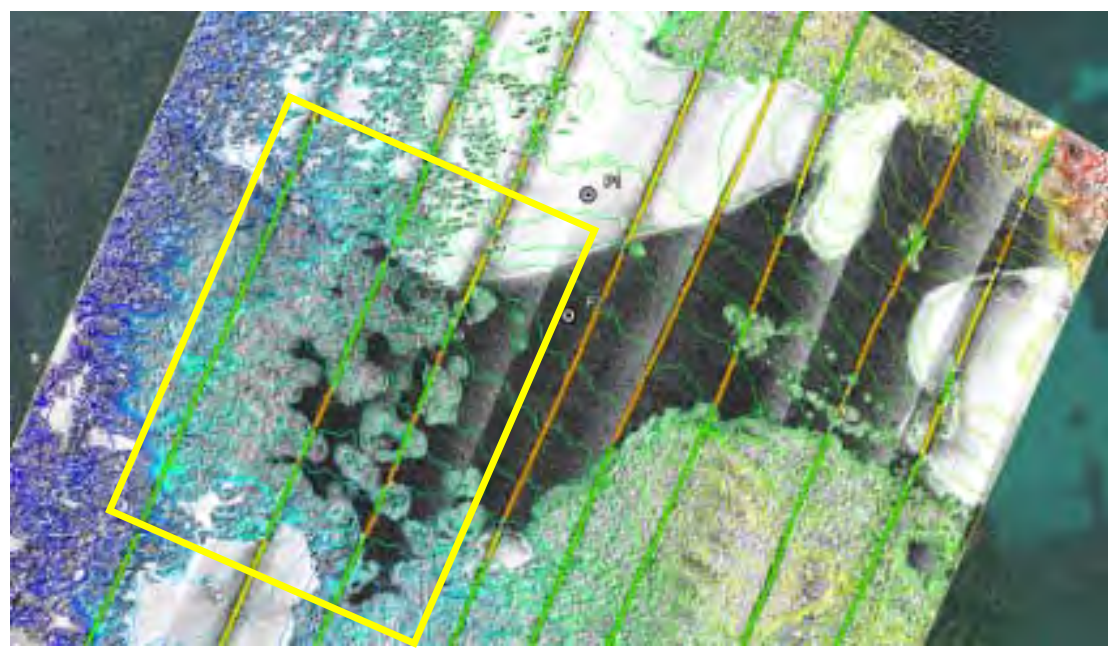

Figure 5. Résultat de la fusion multi-capteurs : superposition de la topographie du fond (imagerie sonar latéral) et de la micro-rugosité surfacique (isobathes fines) définissant le contour de l'aire de présence des végétations sous-marines issue du sondeur multi-

faisceaux ainsi que la classification SACLAF des données sondeur mono-faisceau (Vert : végétation - Orange : sable grossier - Jaune : sable fin). Zone de 500m par $400 \mathrm{~m}$. 


\section{XIV ${ }^{\text {èmes }}$ Journées Nationales Génie Côtier - Génie Civil \\ Toulon, 29 juin au $1^{\text {er }}$ juillet 2016}

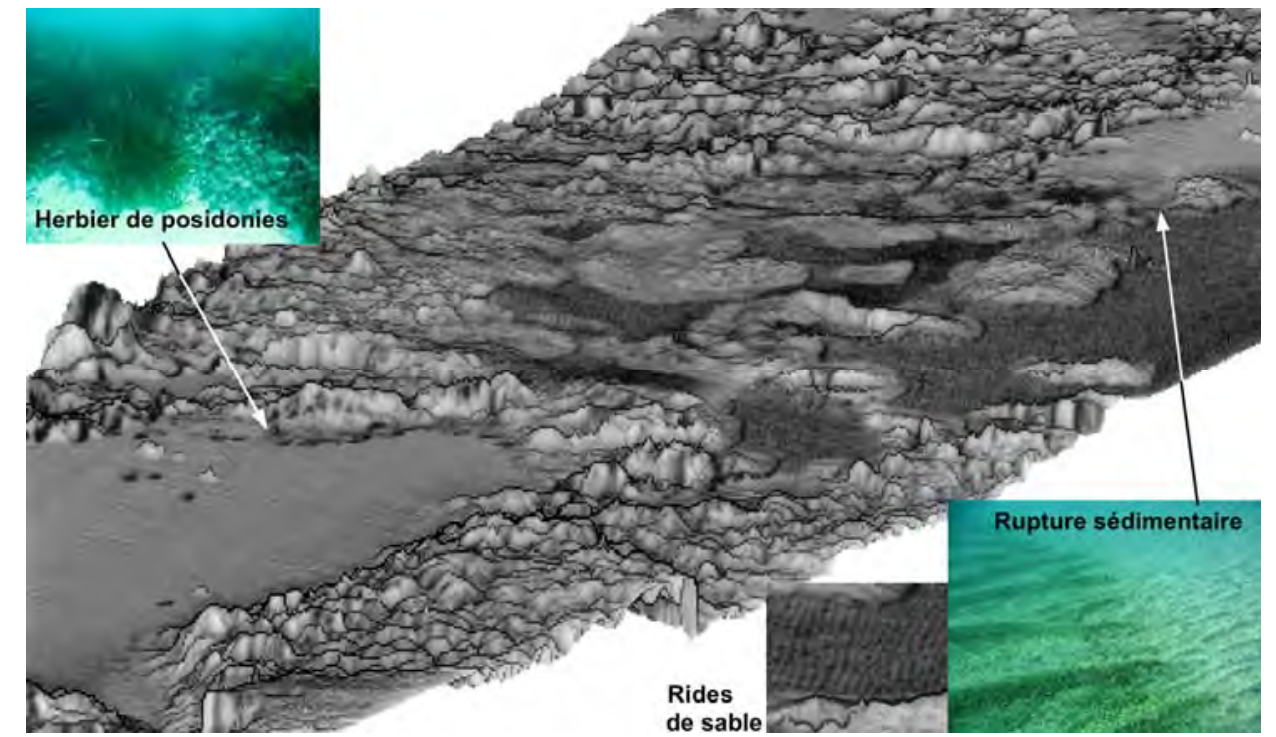

Figure 6. Agrandissement de la figure précédente. Zone de $200 \mathrm{~m}$ par $300 \mathrm{~m}$.

\subsection{Performances de la méthode de fusion}

Enfin les illustrations suivantes (voir figure 7) permettent d'illustrer les performances de la méthode mise au point par comparaison avec les résultats cartographiques des méthodes classiques issues du traitement de données de sonar latéral.
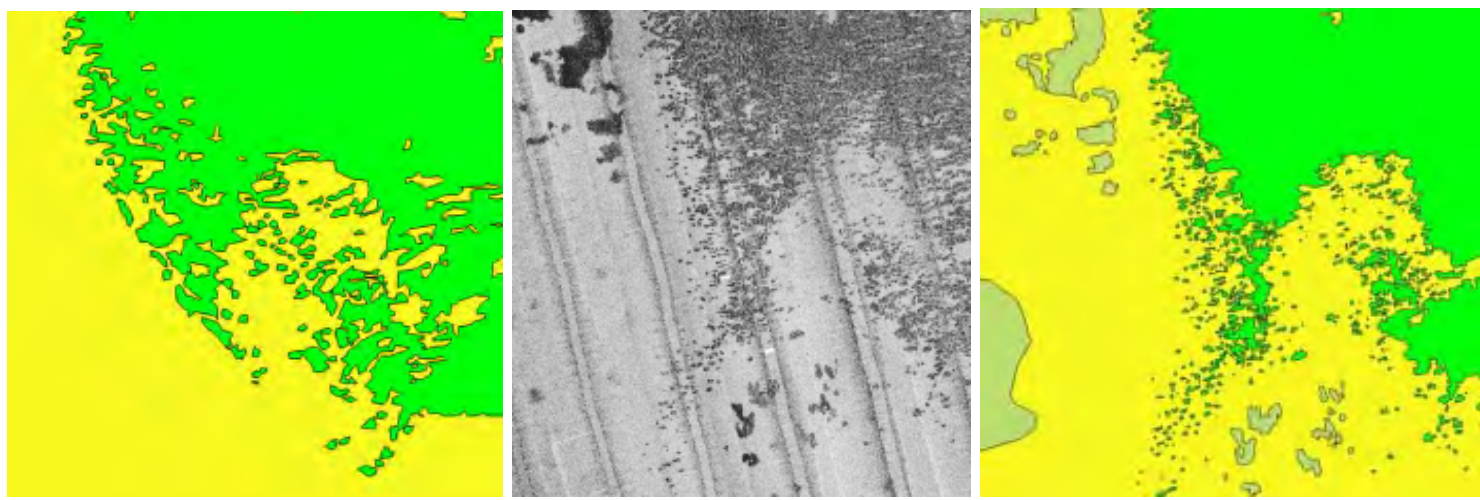

Figure 7. De gauche à droite : Cartographie biocénotique (Méthode habituelle par sonar latéral) - Imagerie sonar latéral - Cartographie issue de la méthode de fusion multi-capteurs. Taille des zones (carrés de 300 m de côté).

En jaune : sable. En vert : Posidonies. En beige : zostères et cymodocées.

La fusion des données permet de mettre en évidence des zones de posidonies (zones représentées en vert) mieux définies, c'est-à-dire pour lesquelles les contours sont plus détaillés. Disposer d'une meilleure résolution permet de mieux appréhender les modifications du milieu (recul de la limite des herbiers) et d'ajuster au mieux les mesures de gestion. En permettant d'augmenter la résolution des cartographies 
biocénotiques et sédimentaires en sortie de traitement des données, donc sans coût supplémentaire, la méthode montre sa haute performance dans le cadre de l'amélioration de la connaissance et du suivi du milieu marin.

Notons de plus que des zones couvertes par d'autres espèces de végétations peuvent aussi être relevées.

\section{Conclusions}

Les méthodes de cartographie basées sur la télédétection acoustique permettent des approches surfaciques très précises (géo-référencement précis de la donnée), très résolvantes (les images admettent des pixels de faible taille) et présentant une grande couverture (les systèmes sont capables en une passe d'acquérir des données sur une large surface). Le développement récent de méthodes et plates-formes instrumentales acoustiques légères en permettent l'utilisation à des coûts désormais accessibles au monde civil pour la gestion de territoires aquatiques et marins. (NOEL et al., 2015)

Ces méthodes offrent des perspectives dans le domaine du suivi ou du monitoring du milieu marin. Présentant des hautes précisions et résolutions et surtout une forte répétitivité (testée in situ sur des zones pilotes pour des périodes de 4 ans), elles permettent d'analyser les évolutions fines du milieu, et de les corréler rapidement aux impacts anthropiques, afin d'ajuster de façon réactive et pertinente les actions de gestion aux impacts observés.

SEMANTIC TS remercie la DS4/MRIS de la DGA et l'Agence de l'eau RMC qui soutiennent ces travaux de monitoring acoustique du milieu marin.

\section{Références bibliographiques}

CHIVERS R.C., EMERSON N., BURNS D.R. (1990). New acoustic processing for underway surveying. The Hydrographic Journal n56, pp 8-17.

NOEL C., VIALA C., LEHN E., JAUFFRET C. (2005). Développement d'une méthode acoustique de détection des herbiers de posidonies. Colloque : Sciences et technologies marines du futur - Un enjeu pour la Méditerranée, Marseille Hotel de Région. 19-20 Mai 2005

NOEL C., MARCHETTI S., BAUER E., TEMMOS JM., COQUET M., BLOUET S., DUPUY DE LA GRANDRIVE R., CHERE E., BOISSERY P., TAFFORIN Y. (2015). Cartographie $3 D$ et surveillance des fonds par fusion multi-capteurs. Application au Coralligène de plateau. merIGéo : De la côte à l'océan : l'information géographique en mouvement. Brest, Ifremer - 24-26 Novembre 2015. 